\title{
Effect of antisense TIMP-1 cDNA on the expression of TIMP-1 and MMP-2 in lung tissue with pulmonary fibrosis induced by bleomycin
}

\author{
HAIYING TANG $^{1 *}$, JINGWEI MAO $^{2 *}$, LILI GAO $^{1}$, JIA LIU $^{1}$ and TAIHUA WU ${ }^{1}$ \\ Departments of ${ }^{1}$ Respiratory Medicine and ${ }^{2}$ Gastroenterology, The First Affiliated Hospital \\ of Dalian Medical University, Dalian, Liaoning 116011, P.R. China
}

Received June 17, 2012; Accepted October 10, 2012

DOI: $10.3892 / \mathrm{mmr} .2012 .1140$

\begin{abstract}
The aim of this study was to observe the effect of antisense tissue inhibitor of matrix metalloproteinase-1 (TIMP-1) cDNA on the concentration of hydroxyproline (HYP) and the expression of TIMP-1 and matrix metalloproteinase-2 (MMP-2) in the lung tissue of rats with bleomycin (BLM)-induced pulmonary fibrosis. Sprague-Dawley rats were randomly divided into 5 groups: the control, pulmonary fibrosis model, sense TIMP-1 transfection, antisense TIMP-1 transfection and empty vector transfection groups. For the transfection groups, following the intratracheal injection of BLM on days 1, 3, 7, 14, 28 and 60 , the rats were treated with retroviral vectors and sacrificed on day 28 . The control and pulmonary fibrosis groups were treated with normal saline at the same time-points. The concentration of HYP and the expression levels of TIMP-1 and MMP-2 in the lung tissue were detected. The HYP concentration and lung tissue TIMP-1 expression levels of the antisense TIMP-1 group decreased significantly on days 1 and 3 compared with those of the empty vector and pulmonary fibrosis groups at the same time-points $(P<0.01)$, but increased significantly in the sense TIMP-1 group $(\mathrm{P}<0.01)$. No significant differences were observed in the HYP concentration and TIMP-1 expression levels in the antisense TIMP-1, sense TIMP-1, empty vector and pulmonary fibrosis groups on days 7, 14, 28 and 60. The lung expression levels of MMP-2 in all groups, with the exception of the control group, had no significant differences at all time-points $(\mathrm{P}>0.05)$. Antisense TIMP-1 cDNA retroviral
\end{abstract}

Correspondence to: Professor Taihua Wu, Department of Respiratory Medicine, The First Affiliated Hospital of Dalian Medical University, 222 Zhongshan Road, Dalian, Liaoning 116011, P.R. China E-mail: wutaihua@sina.com

*Contributed equally

Key words: pulmonary fibrosis, tissue inhibitors of matrix metallo proteinase-1, antisense tissue inhibitors of matrix metalloproteinase-1, matrix metalloproteinase-2 vectors are able to suppress the development of pulmonary fibrosis in the early stages.

\section{Introduction}

Pulmonary fibrosis has an aggressive course and is usually fatal an average of 3 to 6 years after the onset of symptoms. Pulmonary fibrosis refers to a group of lung diseases characterized by inflammation, fibroblast proliferation and excessive collagen deposition. Although the mechanisms underlying pulmonary fibrosis are not clearly understood, current evidence suggests that it is associated with the deposition of extracellular matrix (ECM) components in the lung interstitium (1).

Matrix metalloproteinases (MMPs) are a major group of proteinases known to regulate ECM remodeling and so they are hypothesized to be important in the process of lung fibrosis (2). MMPs and its specific tissue inhibitors of metalloproteinases (TIMPs) have been shown to participate in the parenchymal destruction and repair processes that result in ECM remodeling (3-5). The balance between MMPs and TIMPs is critical in tissue repair and remodeling, and its homeostasis is important in the breakdown and deposition of ECM in the lung (6). Increasing TIMP expression or activation may strengthen the inhibitory effect on MMPs and induce excessive ECM deposition. Conversely, through decreasing lung TIMP expression or activation, excessive ECM may be degraded by increased MMPs, which may relieve pulmonary fibrosis.

Among the MMPs, MMP-2, preferentially secreted by fibroblasts and epithelial cells and mainly suppressed by TIMP-1, has been reported to possess substrate specificity for type IV collagen and is able to degrade basement membrane structures via collagenolytic actions (7).

Thus, after using the antisense TIMP-1 gene to transfect rats with pulmonary fibrosis induced by bleomycin (BLM), we observed the degree of lung fibrosis and lung tissue expression of MMP-2 to investigate the protective role of antisense TIMP-1 gene therapy.

\section{Materials and methods}

Materials. Healthy male Sprague-Dawley (SD) rats weighing 180-220 $\mathrm{g}$ and aged 4-8 weeks were supplied by the specific 
pathogen-free (SPF) Laboratory Animal Center of Dalian Medical University, Dalian, China. Sense and antisense TIMP-1 cDNA retroviral vectors and empty vector were supplied by the Central Laboratory of the First Affiliated Hospital of Dalian Medical University. BLM was purchased from Nippon Shinyaku Co., Ltd. (Kyoto, Japan). TIMP-1 and MMP-2 polyclonal antibodies were supplied by NeoMarkers Inc. (Fremont, CA, USA). The HYC test kit was from Nanjing JianCheng Bioengineering Institute (Nanjing, China). The MaxPoly Plus anti-mouse/rabbit horseradish peroxidase IHC kit was supplied by Fujian Maixin Biological Technology Co. (Fuzhou, China). Primers, DNA markers (DL2000) and the Takara RNA polymerase chain reaction (PCR) 3.0 (AMV) kit were from Takara Biotechnology (Dalian) Co., Ltd. (Dalian, China).

Animal treatment. A total of 180 SD rats were randomly divided into 5 groups: the control, pulmonary fibrosis model, sense TIMP-1 transfection, antisense TIMP-1 transfection and empty vector transfection groups. The SD rat model of pulmonary fibrosis was established by administering a $0.4 \mathrm{mg} \%$ BLM saline injection $(5 \mathrm{mg} / \mathrm{kg})$ by intratracheal injection. For the transfection groups, following the intratracheal injection of BLM on days 1, 3, 7, 14, 28 and 60 , the rats were treated once with retroviral vectors. The control group was subjected to intratracheal injection of normal saline. The pulmonary fibrosis model group was subjected to intratracheal injections of BLM and normal saline at the same time-points as the transfection groups. Rats from all groups, 6 rats per group, were sacrificed on day 28 after the first intratracheal injection. Right lung tissue was collected for reverse transcriptionpolymerase chain reaction (RT-PCR) and hydroxyproline (HYP) concentration assays and left lung tissue was collected for immunohistochemical analysis. The rats were kept in a normally controlled breeding room with standard laboratory food and water for one week prior to the experiments. The rats were maintained in accordance with internationally accepted principles for laboratory animal use.

HYP concentration test. A 1-g sample of right lung tissue was added to $9 \mathrm{ml} 0.86 \%$ saline to prepare a lung tissue homogenate and its supernate was collected for a HYP concentration assay according to the manufacturer's instructions.

TIMP-1 and MMP-2 mRNA expression. mRNA was extracted from the lung tissue samples using TRIzol according to the instructions of the manufacturer (Invitrogen, Carlsbad, CA, USA) and RT-PCR was performed according to the instructions of the RNA PCR 3.0 (AMV) kit. An equal amount of cDNA from each sample was amplified using primers specific to each gene (Table I). DNA amplification was performed using a thermocycler under the following conditions: for TIMP-1, 30 cycles of denaturation at $94^{\circ} \mathrm{C}$ for $60 \mathrm{sec}$, annealing at $51^{\circ} \mathrm{C}$ for $60 \mathrm{sec}$ and extension at $72^{\circ} \mathrm{C}$ for $90 \mathrm{sec}$; for MMP-2, 30 cycles of denaturation at $94^{\circ} \mathrm{C}$ for $45 \mathrm{sec}$, annealing at $55^{\circ} \mathrm{C}$ for $45 \mathrm{sec}$ and extension at $72^{\circ} \mathrm{C}$ for $60 \mathrm{sec}$; and for $\beta$-actin, 30 cycles of denaturation at $94^{\circ} \mathrm{C}$ for $30 \mathrm{sec}$, annealing at $55^{\circ} \mathrm{C}$ for $30 \mathrm{sec}$ and extension at $72^{\circ} \mathrm{C}$ for $60 \mathrm{sec}$. The RT-PCR products were measured by photodensitometry using a gel image analysis system following agarose gel electrophoresis and ethidium bromide staining.
Table I. Oligonucleotide primers of target genes.

\begin{tabular}{lccc}
\hline $\begin{array}{l}\text { mRNA } \\
\text { species }\end{array}$ & Primer sequence (5'-3') & $\begin{array}{c}\text { PCR } \\
\text { product }\end{array}$ \\
\hline TIMP-1 & S & TTTGCATCTCTGGCCTCTG & $495 \mathrm{bp}$ \\
& A & AATGACTGTCACTCTCCAG & \\
MMP-2 & S & CCACATTCTGGCCTGAGCTCCC & $436 \mathrm{bp}$ \\
& A & GATTTGATGCTTCCAAACTTCAC & \\
\multirow{3}{*}{-actin } & S & CCTTCCTGGGCATGGAGTCCTG & $205 \mathrm{bp}$ \\
& A & GGAGCAATGATCTTGATCTTC &
\end{tabular}

TIMP-1, tissue inhibitor of matrix metalloproteinase-1; MMP-2, matrix metalloproteinase-2; $\mathrm{S}$, sense; $\mathrm{A}$, antisense.

Measurement of TIMP-1 and MMP-2 protein expression. Immunohistochemical analysis was carried out according to the Max Vision kit instructions. Image analysis software (Image-pro plus 6.0) was used to measure the light density of the positive control cells in which the cytoplasms were tan-yellow or brown following 3,3'-diaminobenzidine (DAB) staining. For each section, the positive integrated optical density (IOD) and total area of 5 representative visual fields without overlap were observed under a high-powered microscope $(x 400)$. The ratio of IOD and total area represents the mean value of optical density, with a higher ratio indicating a higher level of protein expression.

Statistical analysis. All data were analyzed using the SPSS statistical package version 11.5. The data showed a normal distribution and are expressed as the means \pm standard deviation. The responses of the different experimental groups were analyzed using one-way ANOVA. $\mathrm{P}<0.05$ was considered to indicate a statistically significant result.

\section{Results}

Results of lung tissue HYP concentration assays. The HYP concentrations in the lung tissue of the antisense TIMP-1 group decreased significantly on days 1 and 3 compared with those of the empty vector and pulmonary fibrosis groups at the same time-points $(\mathrm{P}<0.01)$, but increased significantly in the sense TIMP-1 group $(\mathrm{P}<0.01)$. No significant difference was observed in the HYP concentrations of the antisense TIMP-1, sense TIMP-1, empty vector and pulmonary fibrosis groups on days 7, 14, 28 and 60 (Table II).

Results of RT-PCR analysis. The lung TIMP-1 mRNA expression levels of the antisense TIMP-1 group were significantly lower on days 1 and 3 than those of the empty vector and pulmonary fibrosis groups $(\mathrm{P}<0.01)$, but in the sense TIMP-1 group, the TIMP-1 mRNA expression levels were significantly increased $(\mathrm{P}<0.01)$. No significant differences were observed between the TIMP-1 mRNA expression levels in the antisense TIMP-1, sense TIMP-1, empty vector and pulmonary fibrosis groups on days 7, 14, 28 and 60 , or between the lung mRNA expression level of MMP-2 in all groups, with the exception of the control group ( $\mathrm{P}>0.05$; Table III). 
Table II. Lung tissue HYP concentration (mg; mean \pm SD).

\begin{tabular}{|c|c|c|c|c|c|c|}
\hline Group & 1 day & 3 days & 7 days & 14 days & 28 days & 60 days \\
\hline Control & $0.431 \pm 0.460$ & $0.450 \pm 0.054$ & $0.481 \pm 0.060$ & $0.502 \pm 0.086$ & $0.422 \pm 0.058$ & $0.432 \pm 0.048$ \\
\hline Pulmonary fibrosis & $1.152 \pm 0.157$ & $1.221 \pm 0.172$ & $1.083 \pm 0.156$ & $1.182 \pm 0.129$ & $0.973 \pm 0.148$ & $1.012 \pm 0.170$ \\
\hline Empty vector & $1.224 \pm 0.197$ & $1.182 \pm 0.162$ & $1.110 \pm 0.149$ & $1.243 \pm 0.139$ & $1.110 \pm 0.135$ & $1.173 \pm 0.847$ \\
\hline Antisense TIMP-1 & $0.723 \pm 0.153^{\mathrm{a}}$ & $0.642 \pm 0.107^{\mathrm{a}}$ & $0.961 \pm 0.188$ & $1.104 \pm 0.243$ & $1.140 \pm 0.129$ & $1.204 \pm 0.200$ \\
\hline Sense TIMP-1 & $1.712 \pm 0.231^{\mathrm{a}}$ & $1.830 \pm 0.210^{\mathrm{a}}$ & $1.224 \pm 0.193$ & $1.202 \pm 0.177$ & $1.134 \pm 0.177$ & $1.184 \pm 0.216$ \\
\hline
\end{tabular}

HYP, hydroxyproline; TIMP-1, tissue inhibitor of matrix metalloproteinase-1; ${ }^{\mathrm{a}}<0.05 \mathrm{vs}$. empty vector and pulmonary fibrosis groups.

Table III. Lung TIMP-1 mRNA expression at different time-points (mean $\pm \mathrm{SD}$ ).

\begin{tabular}{cccccc}
\hline Day & Control group & Fibrosis group & Empty vector group & Antisense group & Sense group \\
\hline 1 & $0.4005 \pm 0.0157$ & $0.7639 \pm 0.0167$ & $0.7589 \pm 0.0187$ & $0.6384 \pm 0.0152^{\mathrm{a}}$ & $0.9032 \pm 0.0208^{\mathrm{a}}$ \\
3 & $0.4035 \pm 0.0147$ & $0.7666 \pm 0.0158$ & $0.7596 \pm 0.0164$ & $0.5760 \pm 0.0015^{\mathrm{a}}$ & $1.4948 \pm 0.0355^{\mathrm{a}}$ \\
7 & $0.4036 \pm 0.0156$ & $0.7687 \pm 0.0473$ & $0.7645 \pm 0.0363$ & $0.7574 \pm 0.0140$ & $0.7560 \pm 0.0195$ \\
14 & $0.4030 \pm 0.0154$ & $0.7665 \pm 0.0101$ & $0.7575 \pm 0.0101$ & $0.7545 \pm 0.0134$ & $0.7599 \pm 0.0129$ \\
28 & $0.4035 \pm 0.0157$ & $0.7436 \pm 0.0114$ & $0.7476 \pm 0.0254$ & $0.7470 \pm 0.0450$ & $0.7446 \pm 0.0185$ \\
60 & $0.4025 \pm 0.0160$ & $0.7440 \pm 0.0113$ & $0.7465 \pm 0.0215$ & $0.7335 \pm 0.02189$ & $0.7364 \pm 0.0941$ \\
\hline
\end{tabular}

TIMP-1, tissue inhibitor of matrix metalloproteinase-1; ${ }^{a} \mathrm{P}<0.05$ vs. fibrosis and empty vector groups.

Table IV. Lung TIMP-1 protein expression on different time-points (mean \pm SD).

\begin{tabular}{cccccc}
\hline Day & Control group & Fibrosis group & Empty vector group & Antisense group & Sense group \\
\hline 1 & $0.0702 \pm 0.0002$ & $0.0783 \pm 0.0008$ & $0.0781 \pm 0.0018$ & $0.0749 \pm 0.0014^{\mathrm{a}}$ & $0.0812 \pm 0.0017^{\mathrm{b}}$ \\
3 & $0.0700 \pm 0.0018$ & $0.0782 \pm 0.0008$ & $0.0780 \pm 0.0006$ & $0.0736 \pm 0.0010^{\mathrm{a}}$ & $0.0853 \pm 0.0013^{\mathrm{b}}$ \\
7 & $0.0707 \pm 0.0002$ & $0.0782 \pm 0.0007$ & $0.0779 \pm 0.0005$ & $0.0773 \pm 0.0010$ & $0.0772 \pm 0.0008$ \\
14 & $0.0768 \pm 0.0002$ & $0.0768 \pm 0.0006$ & $0.0767 \pm 0.0004$ & $0.0762 \pm 0.0002$ & $0.0763 \pm 0.0010$ \\
28 & $0.0708 \pm 0.0019$ & $0.0772 \pm 0.0003$ & $0.0771 \pm 0.0009$ & $0.0768 \pm 0.0003$ & $0.0769 \pm 0.0005$ \\
60 & $0.0707 \pm 0.0002$ & $0.0772 \pm 0.0004$ & $0.0773 \pm 0.0005$ & $0.0766 \pm 0.0005$ & $0.0768 \pm 0.0005$ \\
\hline
\end{tabular}

TIMP-1, tissue inhibitor of matrix metalloproteinase-1; ${ }^{\mathrm{a}}<0.05$ vs. fibrosis and empty vector groups.

Results of immunohistochemical analysis. The lung TIMP-1 protein expression levels of the antisense TIMP-1 group were significantly lower on days 1 and 3 than those of the empty vector and pulmonary fibrosis groups $(\mathrm{P}<0.01)$, but in the sense TIMP-1 group, the protein expression levels of TIMP-1 were significantly increased $(\mathrm{P}<0.01)$. No significant differences were observed between the TIMP-1 protein expression levels in the antisense TIMP-1, sense TIMP-1, empty vector and pulmonary fibrosis groups on days 7, 14, 28 and 60, or between the lung protein expression levels of MMP-2 in all groups, with the exception of the control group ( $\mathrm{P}>0.05$; Table IV; Figs. 1 and 2).

\section{Discussion}

Tissue fibrosis is the result of abnormal responses to organ injury or to irritation and is typically characterized by the hyperproliferation of fibroblasts and excessive ECM synthesis and secretion. In pulmonary fibrosis, evidence suggests that the constitutive activation of collagen-secreting myofibroblast-like cells is ultimately responsible for increasing the quantity and concentration of collagen. These cells are able to synthesize and secrete excessive amounts of ECM components, particularly collagens, leading to increased tissue stiffness and progressive organ dysfunction (8).

MMPs are a major group of proteinases that are known to regulate ECM remodeling and are hypothesized to be important in the process of lung fibrosis. TIMPs control MMP activities and, therefore, minimize matrix degradation (9). The balance between MMPs and TIMPs is critical in tissue repair and remodeling, and its homeostasis is important in the breakdown and deposition of ECM in the lung. Among the MMPs, MMP-2, preferentially secreted by fibroblasts and epithelial 


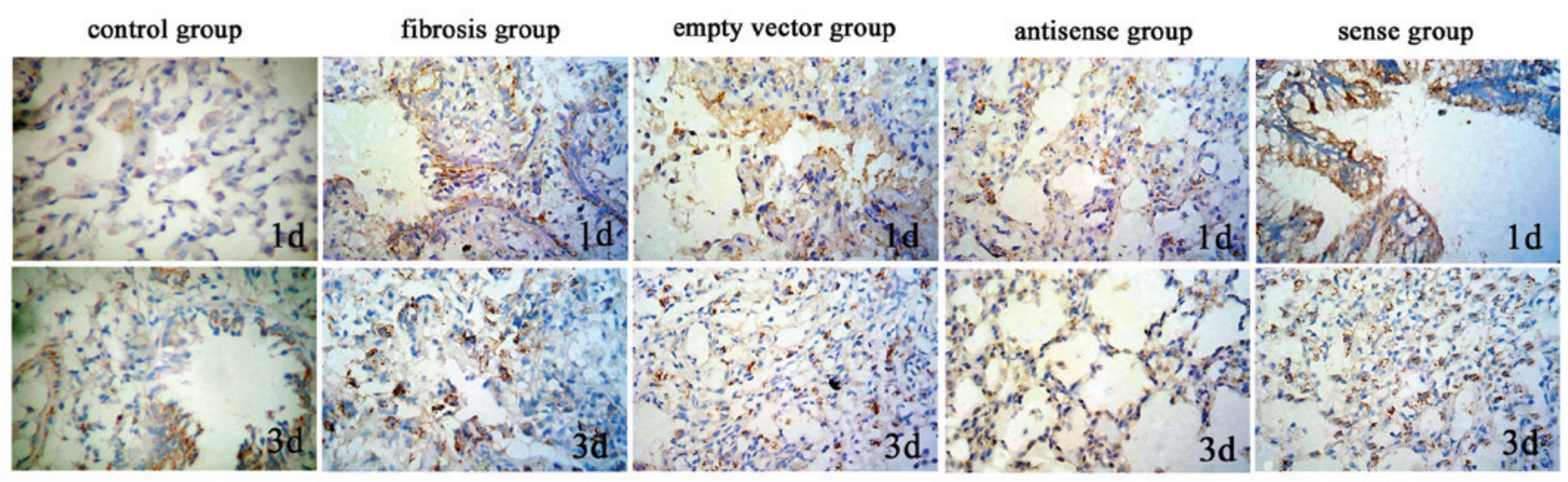

Figure 1. Lung protein expression of tissue inhibitor of matrix metalloproteinase-1 (TIMP-1) on days 1 and 3 in the control, pulmonary fibrosis, empty vector, antisense TIMP-1 and sense TIMP-1 groups. The lung TIMP-1 protein expression levels of the antisense TIMP-1 group were significantly lower on days 1 and 3 than those of the empty vector and pulmonary fibrosis groups $(\mathrm{P}<0.01)$, whereas in the sense TIMP-1 group, the protein expression levels of TIMP-1 increased significantly $(\mathrm{P}<0.01)$.

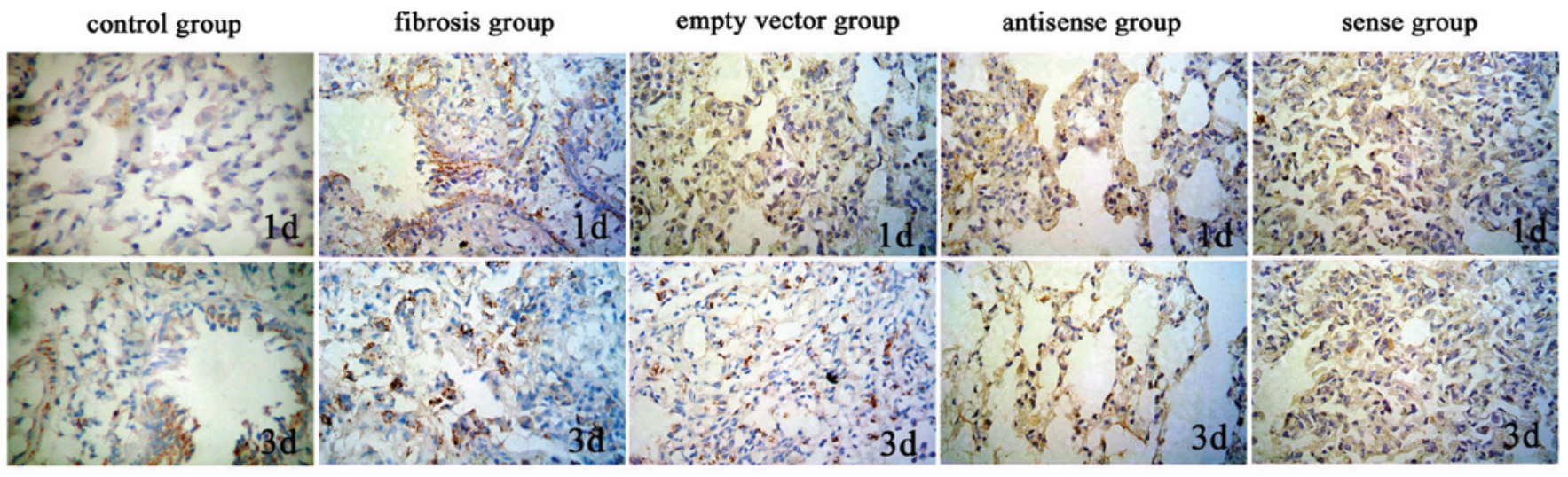

Figure 2. Lung protein expression of matrix metalloproteinase-2 (MMP-2) on days 1 and 3 in the control, pulmonary fibrosis, empty vector, antisense tissue inhibitor of matrix metalloproteinase-1 (TIMP-1) and sense TIMP-1 groups. No significant differences were observed in the lung protein expression levels of MMP-2 in all groups, with the exception of the control group, on days 1 and 3 ( $\mathrm{P}>0.05)$.

cells and mainly suppressed by TIMP-1, has been reported to possess substrate specificity to type IV collagen and is able to degrade basement membrane structures via collagenolytic actions (7).

In our preliminary study, in vitro, sense and antisense TIMP-1 cDNA retroviral vectors were used to transfect mouse fibroblast cells in order to investigate the relationship between pulmonary fibrosis and TIMP-1. The results demonstrated that antisense TIMP-1 cDNA was able to decrease endogenic TIMP-1 expression, increase MMPs activation and then restrain the proliferation of fibroblast cells and reduce the ECM components (10). Based on the above research, in the current study, we investigated the role of antisense TIMP-1 cDNA in pulmonary fibrosis in vivo by observing the effect of antisense TIMP-1 cDNA on the concentration of HYP and the expression of TIMP-1 and MMP-2 in the lung tissue of rats with pulmonary fibrosis induced by BLM.

HYP is a type of collagen having an unusual amino acid composition, the level of which indicates the total content of collagen and the degree of tissue or organ fibrosis. In our study, the HYP concentrations of the antisense TIMP-1 group decreased significantly on days 1 and 3 compared with those of the empty vector and pulmonary fibrosis groups at the same time-points, but increased significantly in the sense TIMP-1 group. This indicates that the use of antisense TIMP-1 cDNA to transfect rats with BLM-induced pulmonary fibrosis relieves lung tissue fibrosis on days 1 and 3, while sense cDNA aggravates fibrosis at the same time-points, in accordance with the pathological changes in the lung tissue.

Pulmonary fibrosis is characterized by the excessive deposition of ECM in the interstitium resulting in respiratory failure. The turnover of ECM is partially regulated by proteases, including MMPs and their inhibitors. Previous studies have demonstrated that the high expression levels of TIMP-1 and low expression levels or weakened activity of MMPs in lung tissue result in pulmonary fibrosis (11-14).

The expression levels of TIMP-1 and MMP-2 in the lung tissue were detected at the mRNA and protein levels in order to investigate the mechanism of the ECM changes in pulmonary fibrosis following transfection by sense and antisense TIMP-1.

In the present study, no significant differences were observed between the lung mRNA and protein expression levels of MMP-2 in the sense- and antisense-transfected groups at all time-points. The results demonstrated that TIMP-1 pref- 
erentially reduced MMP-2 activity rather than expression to degrade ECM following transfection with antisense TIMP-1 cDNA.

We found that the TIMP-1 mRNA and protein expression levels in the lungs of the antisense TIMP-1 group were significantly reduced on days 1 and 3 compared with those of the pulmonary fibrosis group, and in the sense TIMP-1 group, the TIMP-1 expression level increased significantly at these time-points. However, no significant differences were observed in the TIMP-1 expression levels in the antisense and sense TIMP-1 groups on days 7, 14, 28 and 60. It is well known that the cell division of fibroblast cells increases in the early stages of pulmonary fibrosis. Gene carriers, including retroviruses, only transfect cells at the mitotic phase (15), therefore the expression of TIMP-1 in the lung tissue of the antisense TIMP-1 group decreased on days 1 and 3. In the later stage of fibrosis, no significant therapeutic effect of the antisense TIMP-1 cDNA was observed due to low or no transfection efficiency, resulting from a decrease in the number of cells at the mitotic phase, which means that antisense TIMP-1 cDNA retroviral vector transfection has no ability to reverse the formation of pulmonary fibrosis. The results showed that TIMP-1 expression levels increased following transfection with antisense TIMP-1 compared with those of the controls, which suggests that the progress of lung fibrosis is regulated by several factors; growth factors, cytokines, chemokines and regulators of apoptosis have all been implicated in its progression (16)

In conclusion, lung fibrosis induced by BLM may be suppressed by transfection with antisense TIMP-1 cDNA retroviral vectors following the intratracheal injection of BLM on days 1 and 3. Moreover, the observation emphasizes that effective therapies for pulmonary fibrosis must be given in the early stages of the disease, prior to the development of expansive lung destruction and fibrosis.

\section{Acknowledgements}

The authors thank the Central Laboratory of The First Affiliated Hospital of Dalian Medical University. We also thank the SPF Laboratory Animal Center of Dalian Medical University. The study was supported by grants from the National Natural Science Foundation of China (NSFC; 30370620).

\section{References}

1. Suga M, Iyonaga K, Okamoto T, Gushima Y, Miyakawa $H$, Akaike T and Ando M: Characteristic elevation of matrix metalloproteinase activity in idiopathic interstitial pneumonias. Am J Respir Crit Care Med 62: 1949-1956, 2000.
2. Lagente V, Manoury B, Nénan S, Le Quément C, MartinChouly $\mathrm{C}$ and Boichot $\mathrm{E}$ : Role of matrix metalloproteinases in the development of airway inflammation and remodeling. Braz $\mathrm{J}$ Med Biol Res 38: 1521-30, 2005.

3. Hayashi T, Stetler-Stevenson WG, Fleming MV, Fishback N, Koss MN, Liotta LA, Ferrans VJ and Travis WD: Immunohistochemical study of metalloproteinases and their tissue inhibitors in the lungs of patients with diffuse alveolar damage and idiopathic pulmonary fibrosis. Am J Pathol 149: 1241-1256, 1996.

4. Fukuda Y, Ishizaki M, Kudoh S, Kitaichi M and Yamanaka N: Localization of matrix metalloproteinases-1, -2 , and -9 and tissue inhibitor of metalloproteinase-2 in interstitial lung diseases. Lab Invest 78: 687-698, 1998.

5. Finlay GA, O'Driscoll LR, Russell KJ, D'Arcy EM, Masterson JB, FitzGerald MX and O'Connor CM: Matrix metalloproteinase expression and production by alveolar macrophages in emphysema. Am J Respir Crit Care Med 156: 240-247, 1997.

6. Suzuki R, Miyazaki Y, Takagi K, Torii K and Taniguchi H: Matrix metalloproteinases in the pathogenesis of asthma and COPD: implications for therapy. Treat Respir Med 3: 17-27, 2004.

7. Cataldo DD, Gueders MM, Rocks N, Sounni NE, Evrard B, Bartsch P, Louis R, Noel A and Foidart JM: Pathogenic role of matrix metalloproteases and their inhibitors in asthma and chronic obstructive pulmonary disease and therapeutic relevance of matrix metalloproteases inhibitors. Cell Mol Biol (Noisy-le-grand) 49: 875-884, 2003.

8. Cox TR and Erler JT: Remodeling and homeostasis of the extracellular matrix: implications for fibrotic diseases and cancer. Dis Model Mech 4: 165-178, 2011.

9. Bourboulia D and Stetler-Stevenson WG: Matrix metalloproteinases (MMPs) and tissue inhibitors of metalloproteinases (TIMPs): Positive and negative regulators in tumor cell adhesion. Semin Cancer Biol 20: 161-168, 2010.

10. Wu TH, Ling HL and Zhu ZM: Effect of sense and antisense TIMP-1 gene to ECM expression of mouse fibroblast cells. Zhong Guo Man Xing Bing Yu Fang Yu Kong Zhi 12: 20-23, 2004 (In Chinese)

11. McKeown S, Richter AG, O'Kane C, McAuley DF and Thickett DR: MMP expression and abnormal lung permeability are important determinants of outcome in IPF. Eur Respir J 33: 77-84, 2009.

12. Manoury B, Nénan S, Guénon I, Lagente V and Boichot E: Influence of early neutrophil depletion on MMPs/TIMP-1 balance in bleomycin-induced lung fibrosis. Int Immunopharmacol 7: 900-911, 2007.

13. Manoury B, Caulet-Maugendre S, Guénon I, Lagente V and Boichot E: TIMP-1 is a key factor of fibrogenic response to bleomycin in mouse lung. Int J Immunopathol Pharmacol 19: 471-487, 2006.

14. Tian XL, Yao W, Guo ZJ, Gu L and Zhu YJ: Low dose pirfenidone suppresses transforming growth factor beta-1 and tissue inhibitor of metalloproteinase-1, and protects rats from lung fibrosis induced by bleomycina. Chin Med Sci J 21: 145-151, 2006.

15. Robbins PD and Ghivizzani SC: Viral vectors for gene therapy. Pharmacol Ther 80: 35-47, 1998.

16. Cook DN, Brass DM and Schwartz DA: A matrix for new ideas in pulmonary fibrosis. Am J Respir Cell Mol Biol 27: 122-124, 2002. 